\title{
Analysis of biological therapeutic agents and biosimilars
}

\author{
Karen Phinney
}

Published online: 5 September 2014

(C) Springer-Verlag Berlin Heidelberg (outside the USA) 2014

Biological therapeutics have been the fastest growing sector of the pharmaceutical market, and these products have already had a significant impact on the treatment of diseases such as cancer, multiple sclerosis, and rheumatoid arthritis. This new generation of blockbuster drugs is frequently produced in living cells rather than through chemical synthesis and has been made possible by advances in biotechnology, including recombinant DNA techniques. Instead of choosing the most cost-effective synthetic pathway, pharmaceutical companies are now engineering cellular expression systems, operating large bioreactors, and performing complex purification steps to isolate the desired product.

Although biopharmaceuticals have offered tremendous hope for treating disease, they are accompanied by manufacturing, analytical, and regulatory challenges not encountered with traditional drugs. Biological therapeutics produced in cells are not a single entity but rather a mixture of species with variable properties that can ultimately affect the safety and efficacy of the end product. Hence, the characterization of these products goes well beyond tests for purity and often incorporates examination of attributes such as higherorder structure, aggregation, sequence and charge variants, and glycosylation. Companies producing biological therapeutics are faced with employing a large number of analytical techniques, including various types of chromatography, electrophoresis, mass spectrometry, and spectroscopy to address the diverse analytical needs and to ensure that changes in an important aspect of the product do not go undetected. Advances in analytical technologies are also expanding the types

Published in the topical collection Analysis of Biological Therapeutic Agents and Biosimilars with guest editor Karen Phinney.

K. Phinney $(\square)$

NIST National Institute of Standards \& Technology, Biomolecular

Measurement Division, 100 Bureau Drive, Stop 8314,

Gaithersburg, MD 20899, USA

e-mail: karen.phinney@nist.gov of data that can be collected for biomolecules. At the same time, understanding what aspects of the product have the greatest impact on safety and efficacy is essential to establishing a robust regulatory framework.

The complexities of production of biological therapeutics coupled with the extensive characterization necessary to monitor critical attributes of the product means that biological therapeutics tend to be much more costly for both the manufacturer and the patient than traditional small molecule drugs. As a result, cost factors can limit accessibility of these new therapeutics. However, the concept of producing generic drugs as mechanism for driving down treatment costs is not feasible in the world of biologics because cellular production methods do not yield a single chemical entity. Instead, companies are pursuing the development of biosimilars, meaning products that are not exact copies of a previously approved product but retain the general characteristics of the original product. The path to regulatory approval of biosimilar products is still being determined and may vary from product to product. Nevertheless, analytical techniques will play an essential role in development and regulatory consideration and approval of these products. This collection of papers focusses on the analytical challenges and potential future developments for the chemical characterization of biological therapeutics and biosimilars.

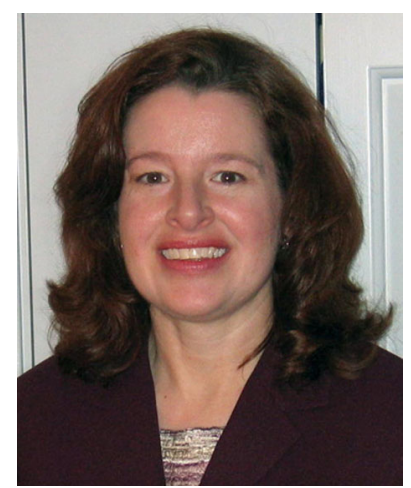

Karen Phinney is a research chemist and group leader at the National Institute of Standards and Technology in Gaithersburg, MD. Her interests include the application of separation techniques and mass spectrometry to protein characterization and quantification. She has been involved in the development of a number of reference materials during her career at NIST and is currently participating in a large, collaborative effort to develop a monoclonal antibody reference material. 